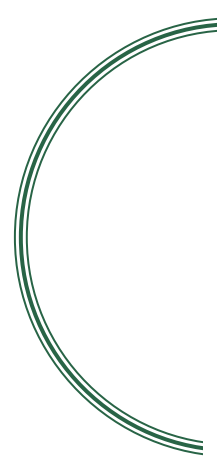

\title{
Immune Checkpoint Blockade Therapy: The 2014 Tang Prize in Biopharmaceutical Science
}

\author{
Ya-Shan Chen ${ }^{1,2}$, Chia-Rui Shen ${ }^{1,2}$
}

The first Tang Prize for Biopharmaceutical Science has been awarded to Prof. James P. Allison and Prof. Tasuku Honjo for their contributions leading to an entirely new way to treat cancer by blocking the molecules cytotoxic T lymphocyte-associated antigen 4 (CTLA-4) and programmed cell death protein 1 (PD-1) that turn off immune response. The treatment, called "immune checkpoint blockade therapy," has opened a new therapeutic era. Here the discoveries of the immune checkpoints and how they contribute to the maintenance of self-tolerance, as well as how to protect tissues from the excess immune responses causing damage are reviewed. The efforts made by Prof. Allison and Prof. Honjo for developing the most promising approaches to activate therapeutic antitumor immunity are also summarized. Since these certain immune checkpoint pathways appear to be one of the major mechanisms resulting in immune escape of tumors, the presence of anti-CTLA-4 and/or anti-PD-1 should contribute to removal of the inhibition signals for T cell activation. Subsequently, it will enhance specific $\mathrm{T}$ cell activation and, therefore, strengthen antitumor immunity. (Biomed J 2015;38:5-8)

Key words: cancer immunotherapy, CTLA-4, immune checkpoint, PD-1, T cells

$\mathrm{T}$ he biennial Tang Prize was established by Taiwan entrepreneur Dr. Samuel Yin in December 2012, and is committed to encourage inquiring minds to explore new perspectives and insights that help to make the world a better place. The Tang Prize is awarded to encourage individuals across the globe to chart the middle path for achieving sustainable development in the four major fields of Sustainable Development, Biopharmaceutical Science, Sinology, and the Rule of Law. The Tang Prize provides each a cash reward of NT $\$ 40$ million, and the research projects proposed by the laureates will also receive a grant of up to NT $\$ 10$ million.
The first Tang Prize in Biopharmaceutical Science has been awarded to Prof. James P. Allison and Prof. Tasuku Honjo for the discovery of cytotoxic T lymphocyte-associated antigen 4 (CTLA-4) and programmed cell death protein 1 (PD-1) as immune inhibitory molecules, ${ }^{[1,2]}$ which led to their applications in cancer immunotherapy. ${ }^{[2-4]}$ In fact, researches on these two immune inhibitory molecules have also demonstrated that CTLA-4 and PD- 1 are the therapeutic targets for allergy, autoimmune and infectious diseases. ${ }^{[2]}$ It indicates that the discovery of CTLA-4 and PD-1 has ushered in a new era for the development of targeting therapy.

From the ${ }^{1}$ Department of Medical Biotechnology and Laboratory Science, College of Medicine, Chang Gung University, Taoyuan, Taiwan; ${ }^{2}$ Graduate Institute of Biomedical Science, College of Medicine, Chang Gung University, Taoyuan, Taiwan

Received: Dec. 19, 2014; Accepted: Jan. 29, 2015

Correspondence to: Dr. Chia-Rui Shen, Department of Medical Biotechnology and Laboratory Science, Chang Gung University. 259 Wenhua $1^{\text {st }}$ Rd., Gueishan, Taoyuan 333, Taiwan (ROC). Tel: 886-3-2118800, ext. 5200;

Fax: 886-3-2118698; E-mail: crshen@mail.cgu.edu.tw

DOI: $10.4103 / 2319-4170.151150$ 
Our immune system evolved to protect us against the attack of foreign microorganisms. One of the key attributes is how the T cells activate and distinguish "self" from "non-self" molecules. Such a critical process involves presentation of antigens to $\mathrm{T}$ cells by antigen presenting cells (APC) and is highly regulated by molecules on T cells and APC. ${ }^{[5]}$ The presence of these molecules, called immune checkpoints, is not only a trigger to sufficient immune response but also to inhibit the stimulation to ensure the inductive immune response is not excessive. In fact, the immune checkpoints, usually referred to as the molecules of inhibitory pathways in the immune system, are crucial for maintaining self-tolerance and modulating the physiological immune responses in the periphery, in order to avoid or minimize the tissue damage from the excess reactions. The first identified immune checkpoints to have such function are the "CD28 receptor family," which are present on T cells and serve as co-stimulatory signal receptors for $\mathrm{T}$ cell activation. ${ }^{[6,7]}$ It includes co-receptors transmitting stimulatory signals (e.g. CD28 $)^{[6,7]}$ and co-receptors transmitting inhibitory signals (e.g. CTLA-4). ${ }^{[6,7]}$ The counterpart (ligand) for CD28 is the "B7 family," containing B7-1 (CD80) and B7-2 (CD86), which are usually present on APC. ${ }^{[8,9]}$ B7-1/ B7-2 not only binds to CD28, which is usually expressed on mature T cells, but also binds to CTLA-4, expressed in low copy number by $\mathrm{T}$ cells only after activation. As mentioned earlier, CD28 appears to be the stimulatory receptor and delivers signals for $\mathrm{T}$ cell activation and survival, while CTLA-4 is the inhibitory receptor and inhibits $\mathrm{T}$ cell responses and regulates peripheral $\mathrm{T}$ cell tolerance. ${ }^{[6,7]}$ Since the independent binding of CD28 and CTLA-4 with B7-1/B7-2 causes B7-1/B7-2 to play a dual role, ${ }^{[8,9]}$ so the B7-1/B7-2:CD28/CTLA-4 pathway is crucial for $\mathrm{T}$ cell activation.

Prof. Allison, the chairman of the University of Texas MD Anderson Cancer Center, is one of the two scientists to identify CTLA-4 as an inhibitory receptor on T cells in $1995^{[6,7]}$ and was the first to recognize it as a potential target for cancer therapy. ${ }^{[6,7]}$ In 1992, Allison demonstrated the importance of CD28-mediated signaling to activate $\mathrm{T}$ cells and prevent $\mathrm{T}$ cell anergy. ${ }^{[10]} \mathrm{He}$ showed that CD28 is a receptor for co-stimulatory signals and is required for optimal induction of T cells. Next, he established the studies and focused on the interaction with B7/CD28/CTLA-4, and suggested that $\mathrm{T}$ cell antigen receptor stimulation is regulated by CD 28 and inhibited by CTLA- 4 that suppresses T cell proliferation and interleukin-2 (IL-2) production. ${ }^{[1]}$ Accordingly, he and his team developed the blockade of CTLA-4 immunotherapy and obtained many encouraging results. ${ }^{[6,7]}$ They used the anti-CTLA-4 and granulocyte/ macrophage colony-stimulating factor (GM-CSF) producing vaccines for murine melanoma $\mathrm{B} 16$, and $80 \%$ of the established tumors could be eradicated. ${ }^{[11]}$ They proved that the CTLA-4 blockade could enhance T cell activation and memory against a highly tumorigenic and poorly immunogenic murine tumor. Although tumor challenge increased the frequency of Tregs, the anti-CTLA- 4 induced T-effector activation and infiltration. Therefore, it appears that the blockade of CTLA-4 changed the balance of Tregs and Teffs and such subsequently resulted in tumor rejection. ${ }^{[12,13]}$ This subsequently led to development of a monoclonal antibody drug, which has undergone clinical trials against stage 4 melanoma and was approved for treatment of melanoma by the US FDA in 2011. ${ }^{[14]}$

In fact, during developing the anti-CTLA-4 therapy, they noted that the surviving mice developed depigmentation in the subcutaneous and metastatic melanoma models indicating that autoimmunity was concurrently induced. ${ }^{[11]}$ However, even with the side effect of treatment, the anti-CTLA-4 is still considered as a powerful therapy accompany with the acceptable autoimmunity risk. Indeed, there were clinical Phase 3 studies using the anti-CTLA-4, like the one with ipilimumab (Yervoy) in patients with metastatic melanoma. ${ }^{[15-18]}$ In the trials, the anti-CTLA-4 therapy has extinguished untreatable late-stage melanoma in $22 \%$ of patients in clinical trials for 3 years or longer. Although some immune-mediated side effects were noted, most of them were of low grade and reversible with early diagnosis and appropriate management. Nonetheless, $10-17 \%$ of patients had shown high-grade or higher severity of immune-related side effects with $2-3 \%$ of these events resulting in death. ${ }^{[19,20]}$

Similar to B7-1/B7-2:CD28/CTLA-4 pathway, PD-L1/ PD-L2: PD-1 pathway also plays a critical role in regulating $\mathrm{T}$ cell activation and tolerance. Dr. Honjo, a professor at Kyoto University's Department of Immunology and Genomic Medicine, discovered the PD-1 in 1992. ${ }^{[21]}$ PD-1 is expressed on activated T cells and up-regulated following T cell reaceptor (TCR)-mediated activation. ${ }^{[2]}$ Dr. Honjo and his group demonstrated that PD-1 is the member of the immunoglobulin gene superfamily and contains an immune receptor tyrosine-based inhibitory motif. ${ }^{[21]}$ The inhibitory function of PD-1 is induced and involved in the classical type of programmed cell death and in the maintenance of self-tolerance by interaction with the specific ligands (PD-L1 and PD-L2) that serves as a negative regulator of immune responses. ${ }^{[21,22]}$ Like CTLA-4, very low levels of PD-1 are sufficient for inhibition of the earliest stages of T cell activation. ${ }^{[23]}$

PD-1 and PD-L1 are increased in tumor microenvironment and inhibit cytokine production to suppress immune responses. ${ }^{[24]} \mathrm{PD}-1 / \mathrm{PD}-\mathrm{L}$ can serve as a potent mechanism for potentially immunogenic tumors to escape from host immune responses. Generally, our immune system is able to recognize cancer cells and attack them in a process called "immune surveillance." However, during tumor development, cancer cells are equipped with machineries 


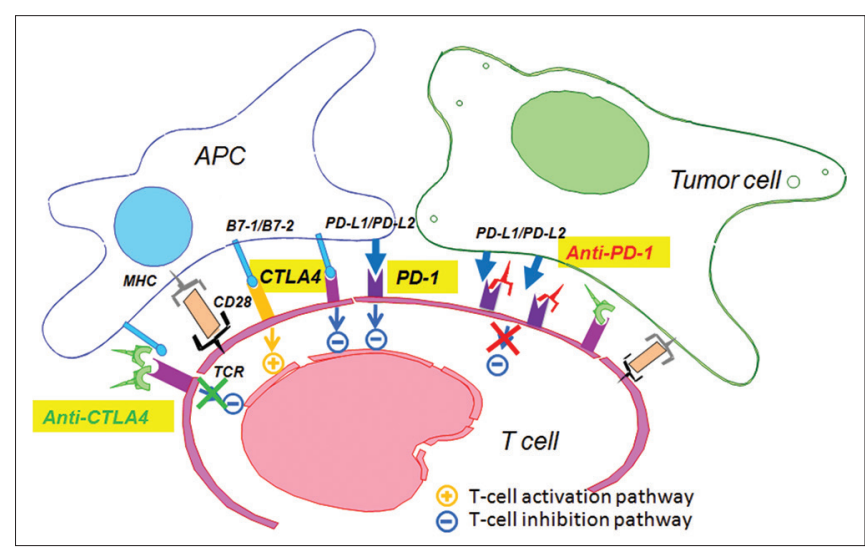

Figure 1: A schematic diagram of $\mathrm{T}$ cells involved in antitumor immunity. It shows the interaction between CD28, CTLA-4, and PD-1 on T cells and their ligands on antigen presenting cells (APC) and tumors. In the presence of anti-CTLA-4 and/or anti-PD-1, the inhibition signals for $\mathrm{T}$ cell activation are blocked and this, in turn, enhances $\mathrm{T}$ cell activation and subsequently strengthens antitumor immunity.

to evade the host antitumor activity, which is described as "immune escape." For example, cancer cells can also express B7 family ligands on their surfaces and, by engaging the co-receptors transmitting inhibitory signals on T cells, they can inhibit the host antitumor $\mathrm{T}$ cell activity. ${ }^{[4,25,26]}$ Dr. Honjo and his team used the blockade of PD-1 in tumor immunotherapy. ${ }^{[4,27]}$ They demonstrated that the anti-PD-L1 antibody significantly inhibited the growth of myeloma cells in mice and the phenomenon could be confirmed in the PD-1-deficient mice. ${ }^{[4]}$ The PD-1 blockade also can be used in the poorly immunogenic B16 melanoma which originally does not express PD-L1. The PD-1 blockade inhibited tumor growth by enhancing recruitment of effector $\mathrm{T}$ cells at tumor sites and prolonged $\mathrm{T}$ cell proliferation and cytokine production. ${ }^{[27]}$ The blockade of interaction between PD-1 and its ligands provides an effective strategy for specific tumor immunity.

Antibodies against PD- 1 have been approved by the US FDA as an investigational new drug and have been developed for the treatment of cancer. Nivolumab is one of the PD-1 antibodies used in the Phase 1 trials which showed that Nivolumab prolonged overall survival, tumor regression, and disease stabilization. The adverse events were mild in most patients. Severe colitis was noted in some patients, but it was infrequent. ${ }^{[28-30]}$ PD-1 blockade is applicable beyond immunogenic tumor types, such as melanoma and renal cell cancer, metastatic non-small-cell lung cancer, a tumor type that has not been considered to be responsive to immunotherapy. Phase 2 trials are ongoing, and Phase 3 studies of anti-PD-1 antibody for the treatment of non-small-cell lung cancer, melanoma, and renal-cell cancer are being planned. ${ }^{[28,29,31]}$

The cancer cells could be recognized by the immune system and the immune system may control or even eliminate tumor cells in some situations. The potential of T cells to amplify antitumor immune responses and destroy tumors has long been recognized. ${ }^{[32]}$ But tumors can enhance immune inhibitory receptors and reduce the immune responses to avoid immune surveillance. ${ }^{[33]}$ Moreover, the immune system can suppress viral infections to protect the host from virus-induced tumors, and also can eliminate the pathogens and promote the prevention of inflammatory environment that could cause tumor development. ${ }^{[34]}$ However, tumor cells can develop various strategies to escape immune surveillance, such as absence of specific tumor antigen, the weak expression of MHC molecules, and recruitment of immune suppressor molecules. ${ }^{[33]}$ Accumulating data suggest that CTLA-4 and PD-1 can raise the threshold of signals needed for effective $\mathrm{T}$ cell proliferation that cause the down-regulation of $\mathrm{T}$ cell response and blockade of this inhibition could enhance the $\mathrm{T}$ cell responses. ${ }^{[11,12,27,35]}$ It indicates that CTLA-4 limits the initiation of T cell activation and clonal expansion, whereas PD-1 contributes to inhibit $\mathrm{T}$ cell function in peripheral tissues [Figure 1]. Thus, the antitumor activity of combined blockade of PD-1 and CTLA- 4 could be more effective, rapid, and produce deeper clinical tumor responses than the blockade of either of them alone. ${ }^{[30,36,37]}$ Therefore, enhancing the T cell response by blocking the inhibitory receptor can be an effective antitumor therapy.

In conclusion, Dr. Allison and Dr. Honjo lead their teams to develop an antibody of these two inhibitory molecules that is used in cancer immunotherapy. The blockade of CTLA-4 and PD-1 shows encouraging antitumor response and manageable adverse events. These results offer new hope for cancer patients.

\section{ACKNOWLEDGMENTS}

The authors declare that they have no conflicts of interest and acknowledge the financial support of grants from the National Science Council (101-2320-B-182-027-MY3) and Chang Gung Memorial Hospital (CMRPD3B0051 and CMRPD1D0031). YSC and CRS contributed equally to this work.

\section{REFERENCES}

1. Krummel MF, Allison JP. CD28 and CTLA-4 have opposing effects on the response of T cells to stimulation. J Exp Med 1995;182:459-65.

2. Okazaki T, Honjo T. PD-1 and PD-1 ligands: From discovery to clinical application. Int Immunol 2007;19:813-24.

3. Leach DR, Krummel MF, Allison JP. Enhancement of antitumor immunity by CTLA-4 blockade. Science 1996;271:1734-6.

4. Iwai Y, Ishida M, Tanaka Y, Okazaki T, Honjo T, Minato N 
Involvement of PD-L1 on tumor cells in the escape from host immune system and tumor immunotherapy by PD-L1 blockade. Proc Natl Acad Sci 2002;99:12293-7.

5. Cantrell DA. T-cell antigen receptor signal transduction. Immunology 2002;105:369-74.

6. Linsley PS, Ledbetter JA. The role of the CD28 receptor during T cell responses to antigen. Annu Rev Immunol 1993;11:191-212.

7. Linsley PS, Clark EA, Ledbetter JA. T-cell antigen CD28 mediates adhesion with $\mathrm{B}$ cells by interacting with activation antigen B7/BB-1. Proc Natl Acad Sci USA 1990;87:5031-5.

8. Greenwald RJ, Freeman GJ, Sharpe AH. The B7 family revisited. Annu Rev Immunol 2005;23:515-48.

9. Sharpe AH, Freeman GJ. The B7-CD28 superfamily. Nat Rev Immunol 2002;2:116-26.

10. Harding FA, McArthur JG, Gross JA, Raulet DH, Allison JP. CD28-mediated signalling co-stimulates murine T cells and prevents induction of anergy in T-cell clones. Nature 1992;356:607-9.

11. van Elsas A, Hurwitz AA, Allison JP. Combination immunotherapy of B16 melanoma using anti-cytotoxic T lymphocyte-associated antigen 4 (CTLA-4) and granulocyte/macrophage colony-stimulating factor (GM-CSF)-producing vaccines induces rejection of subcutaneous and metastatic tumors accompanied by autoimmune depigmentation. J Exp Med 1999;190:355-66.

12. Quezada SA, Peggs KS, Curran MA, Allison JP. CTLA4 blockade and GM-CSF combination immunotherapy alters the intratumor balance of effector and regulatory T cells. J Clin Invest 2006;116:1935-45.

13. Pedicord VA, Montalvo W, Leiner IM, Allison JP. Single dose of anti-CTLA-4 enhances CD8+T-cell memory formation, function, and maintenance. Proc Natl Acad Sci 2011;108:266-71.

14. Mansh M. Ipilimumab and cancer immunotherapy: A new hope for advanced stage melanoma. Yale J Biol Med 2011;84:381-9.

15. Hodi FS, O'Day SJ, McDermott DF, Weber RW, Sosman JA, Haanen JB, et al. Improved survival with ipilimumab in patients with metastatic melanoma. N Engl J Med 2010;363:711-23.

16. Robert C, Thomas L, Bondarenko I, O’Day S, Weber J, Garbe C, et al. Ipilimumab plus dacarbazine for previously untreated metastatic melanoma. N Engl J Med 2011;364:2517-26.

17. McDermott D, Haanen J, Chen TT, Lorigan P, O'Day S, Investigators MDX. Efficacy and safety of ipilimumab in metastatic melanoma patients surviving more than 2 years following treatment in a phase III trial (MDX010-20). Ann Oncol 2013;24:2694-8.

18. Kitano S, Tsuji T, Liu C, Hirschhorn-Cymerman D, Kyi C, Mu Z, et al. Enhancement of tumor-reactive cytotoxic $\mathrm{CD} 4+\mathrm{T}$ cell responses after ipilimumab treatment in four advanced melanoma patients. Cancer Immunol Res 2013;1:235-44.

19. Fellner C. Ipilimumab (Yervoy) Prolongs Survival In Advanced Melanoma: Serious Side Effects and a Hefty Price Tag May Limit Its Use. P T 2012;37:503-30.

20. Culver ME, Gatesman ML, Mancl EE, Lowe DK. Ipilimumab: A novel treatment for metastatic melanoma. Ann Pharmacother 2011;45:510-9.

21. Ishida Y, Agata Y, Shibahara K, Honjo T. Induced expression of
PD-1, a novel member of the immunoglobulin gene superfamily, upon programmed cell death. EMBO J 1992;11:3887-95.

22. Nishimura H, Nose M, Hiai H, Minato N, Honjo T. Development of Lupus-like Autoimmune Diseases by Disruption of the PD-1 Gene Encoding an ITIM Motif-Carrying Immunoreceptor. Immunity 1999; 11:141-51.

23. Chemnitz JM, Parry RV, Nichols KE, June CH, Riley JL. SHP-1 and SHP-2 associate with immunoreceptor tyrosine-based switch motif of programmed death 1 upon primary human T cell stimulation, but only receptor ligation prevents $\mathrm{T}$ cell activation. J Immunol 2004;173:945-54.

24. Hino R, Kabashima K, Kato Y, Yagi H, Nakamura M, Honjo T, et al. Tumor cell expression of programmed cell death-1 ligand 1 is a prognostic factor for malignant melanoma. Cancer 2010;116:1757-66.

25. Dong H, Strome SE, Salomao DR, Tamura H, Hirano F, Flies DB, et al. Tumor-associated B7-H1 promotes T-cell apoptosis: A potential mechanism of immune evasion. Nat Med 2002;8:793-800.

26. Zou W, Chen L. Inhibitory B7-family molecules in the tumour microenvironment. Nat Rev Immunol 2008;8:467-77.

27. Iwai Y, Terawaki S, Honjo T. PD-1 blockade inhibits hematogenous spread of poorly immunogenic tumor cells by enhanced recruitment of effector T cells. Int Immunol 2005;17:133-44.

28. Topalian SL, Hodi FS, Brahmer JR, Gettinger SN, Smith DC, McDermott DF, et al. Safety, Activity, and Immune Correlates of Anti-PD-1 Antibody in Cancer. N Engl J Med 2012;366:2443-54.

29. Brahmer JR, Tykodi SS, Chow LQM, Hwu W-J, Topalian SL, Hwu P, et al. Safety and Activity of Anti-PD-L1 Antibody in Patients with Advanced Cancer. N Engl J Med 2012;366:2455-65.

30. Wolchok JD, Kluger H, Callahan MK, Postow MA, Rizvi NA, Lesokhin AM, et al. Nivolumab plus Ipilimumab in Advanced Melanoma. N Engl J Med 2013;369:122-33.

31. Brahmer JR, Pardoll DM. Immune Checkpoint Inhibitors: Making Immunotherapy a Reality for the Treatment of Lung Cancer. Cancer Immunol Res 2013;1:85-91.

32. Rosenberg SA, Spiess P, Lafreniere R. A new approach to the adoptive immunotherapy of cancer with tumor-infiltrating lymphocytes. Science 1986;233:1318-21.

33. Costello RT, Gastaut JA, Olive D. Tumor escape from immune surveillance. Arch Immunol Ther Exp 1999;47:83-8.

34. Smyth MJ. Tumour immunology. Curr Opin Immunol 2007;19:200-2.

35. Okazaki T, Maeda A, Nishimura H, Kurosaki T, Honjo T. PD-1 immunoreceptor inhibits $\mathrm{B}$ cell receptor-mediated signaling by recruiting src homology 2-domain-containing tyrosine phosphatase 2 to phosphotyrosine. Proc Natl Acad Sci 2001;98:13866-71.

36. Curran MA, Montalvo W, Yagita H, Allison JP. PD-1 and CTLA-4 combination blockade expands infiltrating $\mathrm{T}$ cells and reduces regulatory T and myeloid cells within B16 melanoma tumors. Proc Natl Acad Sci U S A 2010;107:4275-80.

37. Intlekofer AM, Thompson CB. At the Bench: Preclinical rationale for CTLA-4 and PD-1 blockade as cancer immunotherapy. J Leukoc Biol 2013;94:25-39. 